\title{
Genetic Variations of Glutathione S-Transferase Influence on Blood Cadmium Concentration
}

\author{
Nitchaphat Khansakorn, ${ }^{1}$ Waranya Wongwit, ${ }^{1}$ Prapin Tharnpoophasiam, ${ }^{1}$ \\ Bunlue Hengprasith, ${ }^{2}$ Lerson Suwannathon, ${ }^{3}$ Suwannee Chanprasertyothin, ${ }^{4}$ \\ Thunyachai Sura, ${ }^{5}$ Sming Kaojarern, ${ }^{5}$ Piyamit Sritara, ${ }^{5}$ and Jintana Sirivarasai ${ }^{5,6}$ \\ ${ }^{1}$ Department of Social and Environmental Medicine, Faculty of Tropical Medicine, Mahidol University, Bangkok 10400, Thailand \\ ${ }^{2}$ Health Office, Electricity Generating Authority of Thailand, Nonthaburi 11130, Thailand \\ ${ }^{3}$ Excellence Service Center For Medical Technology and Quality Improvement, Faculty of Medical Technology, Mahidol University, \\ Bangkok 10700, Thailand \\ ${ }^{4}$ Office of Research Academic and Innovation, Faculty of Medicine Ramathibodi Hospital, Mahidol University, \\ Bangkok 10400, Thailand \\ ${ }^{5}$ Department of Medicine, Faculty of Medicine Ramathibodi Hospital, Mahidol University, Bangkok 10400, Thailand \\ ${ }^{6}$ Division of Clinical Pharmacology and Toxicology, Department of Medicine, Faculty of Medicine Ramathibodi Hospital, \\ Mahidol University, Bangkok 10400, Thailand
}

Correspondence should be addressed to Jintana Sirivarasai, rajsr@mahidol.ac.th

Received 13 October 2011; Accepted 16 November 2011

Academic Editor: J. J. Stegeman

Copyright (C) 2012 Nitchaphat Khansakorn et al. This is an open access article distributed under the Creative Commons Attribution License, which permits unrestricted use, distribution, and reproduction in any medium, provided the original work is properly cited.

\begin{abstract}
The glutathione S-transferases (GSTs) are involved in biotransformation and detoxification of cadmium (Cd). Genetic polymorphisms in these genes may lead to interindividual variation in Cd susceptibility. The objective of this study was to assess the association of GSTs (GSTT1, GSTM1, and GSTP1 Val105Ile) polymorphisms with blood Cd concentrations in a nonoccupationally exposed population. The 370 blood samples were analyzed for Cd concentration and polymorphisms in GSTs genes. Geometric mean of blood Cd among this population was $0.46 \pm 0.02 \mu \mathrm{g} / \mathrm{L}$ (with $95 \%$ CI; 0.43-0.49 $\mu \mathrm{g} / \mathrm{L}$ ). Blood Cd concentrations in subjects carrying GSTP1 Val/Val genotype were significantly higher than those with Ile/Ile and Ile/Val genotypes. No significant differences in blood Cd concentrations among individual with gene deletions of GSTT1 and GSTM1 were observed. GSTP1/GSTT1 and GSTP1/GSTM1 combinations showed significantly associated with increase in blood Cd levels. This study indicated that polymorphisms of GSTP1 combined with GSTT1 and/or GSTM1 deletion are likely to influence on individual susceptibility to cadmium toxicity.
\end{abstract}

\section{Introduction}

Cadmium (Cd) is widely dispersed in the environment and causes various diseases, both in occupationally exposed individuals and in the general population. The general population may be exposed to cadmium through ingestion of food and drinking water, inhalation of particulates from ambient air or tobacco smoke, or ingestion of contaminated soil or dust. The major long-term toxic effects of low-level cadmium exposure are renal injury, obstructive pulmonary disease, osteoporosis, and cardiovascular disease [1]. Cadmium is also a potent human carcinogen and has been associated with cancers of the lung, prostate, pancreas, and kidney [2].

In human body, Cd is basically taken up by the liver. In the liver, Cd can bind with glutathione (GSH) and be excreted into bile. Moreover, $\mathrm{Cd}$ can bind to metallothionein (MT) and be stored. Some Cd bound to MT leaks into the plasma and then is taken up by the kidney. The balance between CdMT and nonbound Cd in renal tissue may be responsible for the degree of $\mathrm{Cd}$ nephrotoxicity $[3,4]$. Therefore, susceptibility to Cd toxicity among individual may probably be related to genetic variations of Cd-metabolizing enzymes. 
Glutathione S-transferase (GST) gene is one of the potential candidate susceptibility genes-because it plays a significant role in Cd biotransformation and detoxification [5]. The principal function of GST enzymes is conjugations of hydrophobic and electrophilic compounds with reduced glutathione. The intracellular binding reaction with GSH is catalyzed by the GSTs and leads to stable GSH-metal conjugates being transported out of the cell and excreted via feces and urine [6]. Seven GSTs gene families (Alpha, Mu, Pi, Theta, Sigma, Omega, and Zeta) have been described and genetic polymorphisms have been reported for GSTM1, GSTP1, and GSTT1, resulting in either decreased or altered enzyme activity $[7,8]$. GSTT1 and GSTM1 are particularly important, because they have a deletion polymorphism resulting in impaired catalytic activity, which is associated with greater sensitivity to toxic compounds. The homozygous deleted (null genotype) of GSTT1 and GSTM1 genotypes have been associated with enhanced genotoxicity and are believed to be key factors in determining susceptibility to diseases associated with exposure to xenobiotics such as cancer [9]. Polymorphism of GSTP1 has been found in the coding region. The variant allele of GSTP1 Val105Ile encodes valine (Val) instead of isoleucine (Ile) at codon 105 due to a base pair exchange, where $G$ substitutes A. These substitutions were linked to a change in enzyme activity [8]. Polymorphisms in GSTT1, GSTM1, and GSTP1 may be associated with increased susceptibility to cadmium toxicity [10].

Given that metabolism and detoxification of cadmium (as an electrophile) is mediated by glutathione and GSTs are involved in the complexation of electrophilic substances with glutathione, it is reasonable to hypothesize that genetic polymorphisms in GST genes could result in differences in sensitivity to cadmium.

In recent years, the possibilities of applying molecular techniques to toxicogentics are considerably focused, especially for medical investigations and determinations of factors influencing chemical poisonings. These include biochemical and genetic determinants related to acute and chronic effects. To our knowledge, there are limited data on genetic predisposition influence on cadmium detoxification in the human body. Therefore, this study investigated the association of glutathione S-transferase genes (GSTT1, GSTM1, and GSTP1) polymorphisms and blood cadmium concentration in a nonoccupationally exposed population.

\section{Materials and Methods}

2.1. Study Subjects. The Electric Generating Authority of Thailand (EGAT) STUDY was the first cohort study of chronic disease in Thailand, originally designed in 1985 (known as EGAT 1), and mainly covered multidisciplinary researches related to cardiovascular disease (CVD) risk factors such as nutrition and toxicology. The 370 subjects in this study were participants in the third survey of EGAT 2 in 2009 (the first survey started in 1998 and second survey in 2003) [11]. Participants completed a self-administered questionnaire, underwent a physical examination, and provided fasting blood samples. The study was approved by the
Committee on Human Rights Related to Researches Involving Human Subjects, Faculty of Medicine, Ramathibodi Hospital, Faculty of Tropical Medicine, Mahidol University, in accordance with the ethical standards laid down in the 1964 Declaration of Helsinki. All participants gave their informed consent prior to their inclusion in the study.

Toxicological profile of heavy metals was investigated in both first and third surveys of EGAT 2, and further genetic analysis started in 2009. Ten milliliters of blood were collected by venipuncture into EDTA and heparinized tubes from each subject and immediately centrifuged at $2000 \mathrm{~g}$. Buffy coat, erythrocytes, and plasma were separated and stored at $-20^{\circ} \mathrm{C}$ until genotyping analyses and biochemical measurement were performed.

2.2. Determination of Cadmium in Blood. Whole blood cadmium concentrations were measured by graphite furnace atomic absorption spectrometry (GFAAS) with Zeeman background correction after dilution of the blood $(1: 10)$ with Triton X-100 solution containing diammonium hydrogen phosphate and nitric acid [12]. The concentration was expressed as micrograms per liter.

2.3. Genotype Analyses. The genomic DNA was extracted from the lymphocytes by a modified salting out procedure [13] and frozen at $-20^{\circ} \mathrm{C}$ until analysis. The genetic polymorphisms of GSTM1, GSTT1, and GSTP1 were performed by real-time polymerase chain reaction (real-time PCR) according to the method of TaqMan SNP Genotyping Assays on an ABI 7500 instrument (Applied Biosystems, Foster City, CA, USA), in 96-well format. The TaqMan Assay included the forward target-specific polymerase chain reaction (PCR) primer, the reverse primer, and the TaqMan MGB probes labeled with 2 special dyes: FAM and VIC. The concentrations of probes were $0.04 \mu \mathrm{M}$. Amplification of $20 \mathrm{ng}$ of DNA was performed during 40 cycles in a reaction volume of $10 \mu \mathrm{L}$. TaqMan Universal PCR Master Mix was used for analysis. Thermocycling conditions were $95^{\circ} \mathrm{C}$ for 15 seconds follow by $60^{\circ} \mathrm{C}$ for 1 minute. Information of specific probe and primers are available on the National Cancer Institute's SNP500 database web page at http://variantgps.nci.nih.gov/ [14].

2.4. Statistical Analysis. Statistical analyses were carried out using the SPSS 16.0 for window software (SPSS, Inc., Chicaco, IL, USA). Cadmium data were log-transformed to achieve normal distribution. The values were expressed as geometric means. The comparisons between variables were examined by the Student's $t$-test and analysis of variance (ANOVA). Genotype distribution was analyzed with $\chi^{2}$. The effects of all GSTs gene on blood cadmium were evaluated by linear regression analysis. A $P$ value of 0.05 was used as the criterion for statistical significance.

\section{Results}

The subjects of this study consisted of 370 employees of EGAT. The geometric mean of the blood cadmium 
TABLE 1: The geometric mean of blood cadmium concentrations in a nonoccupational exposed population.

\begin{tabular}{lccc}
\hline Characteristics & $n$ & $\mathrm{GM} \pm \mathrm{SE}$ & Blood cadmium concentration $(\mu \mathrm{g} / \mathrm{L})$ \\
$95 \% \mathrm{CI}$ & $0.43-0.49$ \\
\hline $\begin{array}{l}\text { Total } \\
\text { Gender }\end{array}$ & 370 & $0.46 \pm 0.02$ & $0.42-0.50$ \\
$\quad$ male & 267 & $0.46 \pm 0.02$ & $0.40-0.50$ \\
$\quad$ female & 103 & $0.45 \pm 0.03$ & $0.40-0.51$ \\
Age (yrs) & & & $0.42-0.50$ \\
$\quad 45-55$ yrs & 144 & $0.45 \pm 0.03$ & $0.46 \pm 0.02$ \\
$\quad 226$ & & $0.38-0.42$ \\
Smoking habit & & $0.41 \pm 0.02^{\mathrm{a}}$ & $0.62-0.85$ \\
$\quad$ nonsmoker & 294 & $0.72 \pm 0.04$ & \\
$\quad$ Smoker & 76 & & $0.40-0.48$ \\
Alcohol consumption & & $0.44 \pm 0.02$ & $0.43-0.53$ \\
$\quad$ Nondrinker & 178 & $0.47 \pm 0.02$ & \\
$\quad$ Drinker & 192 & & \\
\hline
\end{tabular}

${ }^{a}$ Significantly different from smoker, $P<0.05$ Student's $t$-test.

$\mathrm{GM}=$ Geometric mean.

$95 \% \mathrm{CI}=95 \%$ Confidence interval.

TABLE 2: Geometric means of blood cadmium concentrations in a nonoccupational exposed population categorized by different genotype.

\begin{tabular}{|c|c|c|c|c|}
\hline \multirow{2}{*}{ Gene } & \multirow{2}{*}{ Genotype } & \multicolumn{2}{|c|}{ Frequency } & \multirow{2}{*}{$\begin{array}{c}\text { Blood cadmium }(\mu \mathrm{g} / \mathrm{L}) \\
\mathrm{GM} \pm \mathrm{SE}\end{array}$} \\
\hline & & $n$ & $\%$ & \\
\hline \multirow[t]{2}{*}{ GSTT1 } & Null & 121 & 32.7 & $0.49 \pm 0.03$ \\
\hline & Present & 249 & 67.3 & $0.44 \pm 0.02$ \\
\hline \multirow[t]{2}{*}{ GSTM1 } & Null & 213 & 57.6 & $0.47 \pm 0.02$ \\
\hline & Present & 157 & 42.4 & $0.44 \pm 0.02$ \\
\hline GSTP1-105 & Ile/Ile & 212 & 57.3 & $0.45 \pm 0.02$ \\
\hline \multirow[t]{2}{*}{ rs1695 } & Ile/Val & 139 & 37.6 & $0.45 \pm 0.03$ \\
\hline & $\mathrm{Val} / \mathrm{Val}$ & 19 & 5.1 & $0.71 \pm 0.08^{\mathrm{a}, \mathrm{b}}$ \\
\hline
\end{tabular}

$\overline{\mathrm{a}, \mathrm{b}}$ Significantly different GSTP1 from Ile/Ile and Ile/Val genotypes, respectively, $P<0.05$.

concentrations was $0.46 \pm 0.02 \mu \mathrm{g} / \mathrm{L}$ (with 95\% CI; 0.43$0.49 \mu \mathrm{g} / \mathrm{L}$ ) (Table 1). The level of blood cadmium in smokers was significantly higher than those in nonsmokers $(0.72$ versus $0.42 \mu \mathrm{g} / \mathrm{L}, P<0.05)$. There were no significantly difference in blood cadmium concentration between gender and alcohol consumption.

The distribution of genetic polymorphisms and mean of blood cadmium concentration for each genotype are shown in Table 2. All genes were in Hardy-Weinberg equilibrium $(P$-value $>0.05)$. The GSTT1 present was the most prevalent genotype found in study population (67.3\%). Homozygous variant of GSTP1 Val/Val was the lowest genotype in study population $(5.1 \%)$.

Subjects with GSTP1 Val/Val had significantly higher blood cadmium concentration $(0.71 \mu \mathrm{g} / \mathrm{L})$ than did the GSTP1 wild-type (Ile/Ile) $(0.45 \mu \mathrm{g} / \mathrm{L})$ and heterozygous variant of GSTP1 Ile/Val $(0.45 \mu \mathrm{g} / \mathrm{L}, P<0.05)$. There were no significant differences on blood cadmium concentration among subjects with GSTT1 and GSTM1 deletions.

Three of the putative genes with risk genotypes were examined (Table 3). The linear regression analysis indicated a function of genetic polymorphisms on blood Cd level. In this model, the results of regression coefficient of GSTT1 Val105Ile ( $\beta=0.59, P=0.034)$ exhibited strong potential predictors on blood $\mathrm{Cd}$ concentration. Considering the significant impact of GSTP1 variant allele and the very small number of GSTP1 Val/Val genotype $(n=19)$, we combined these variant-allele subjects with heterozygous genotype $(n=$ 158). Moreover, significant effect of gene combination was observed for GSTP1 variant allele with GSTM1 and GSTT1. Combined GSTP1-105 Ile/Val and Val/Val with GSTM1 and GSTT1 null genotypes were shown as statistical predictors of blood Cd concentrations $(\beta=0.67, P=0.044$ and $\beta=0.72, P=0.038$, resp.). However, no other effects of gene combinations were observed on blood Cd levels.

\section{Discussion}

A nonoccupationally exposed population may be exposed to cadmium through ingestion of food and drinking water and inhalation of particulates from ambient air or tobacco smoke. In the present study, we found that the geometric 
TABLE 3: Regression coefficient for blood cadmium by GSTP1 Val105Ile and interaction between GSTP1 Val105Ile and GSTT1 and GSTM1.

\begin{tabular}{|c|c|c|c|}
\hline \multirow{2}{*}{ Genotype } & \multicolumn{3}{|c|}{ Blood cadmium } \\
\hline & $n$ & 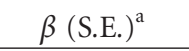 & $P$ value ${ }^{\mathrm{b}}$ \\
\hline \multicolumn{4}{|l|}{ GSTP1 Val105Ile } \\
\hline GSTP1 Ile/Ile & 212 & $0.27(0.19)$ & 0.324 \\
\hline GSTP1 Ile/Val & 139 & $0.35(0.22)$ & 0.296 \\
\hline GSTP1 $\mathrm{Val} / \mathrm{Val}$ & 19 & $0.59(0.39)$ & 0.034 \\
\hline \multicolumn{4}{|l|}{ GSTM1 and GSTP1 Val105Ile } \\
\hline GSTM1 +/GSTP1 Ile/Ile & 96 & $-0.14(0.23)$ & 0.462 \\
\hline GSTM1 +/GSTP1 Ile/Val and Val/Val & 82 & $0.32(0.26)$ & 0.108 \\
\hline GSTM1 -/GSTP1 Ile/Ile & 126 & $0.29(0.17)$ & 0.288 \\
\hline GSTM1 -/GSTP1 Ile/Val and $\mathrm{Val} / \mathrm{Val}$ & 68 & $0.67(0.46)$ & 0.044 \\
\hline \multicolumn{4}{|l|}{ GSTT1 and GSTP1 Val105Ile } \\
\hline GSTT1 +/GSTP1 Ile/Ile & 121 & $0.20(0.13)$ & 0.142 \\
\hline GSTT1 +/GSTP1 Ile/Val and Val/Val & 89 & $0.18(0.21)$ & 0.296 \\
\hline GSTT1-/GSTP1 Ile/Ile & 108 & $0.39(0.22)$ & 0.103 \\
\hline GSTT1 -/GSTP1 Ile/Val and Val/Val & 52 & $0.72(0.58)$ & 0.038 \\
\hline
\end{tabular}

${ }^{a}$ Regression coefficients.

${ }^{\mathrm{b}} P$ value were obtained by linear regression after controlling for sex, age, BMI, smoking status and alcohol consumption.

mean of the blood cadmium concentration in a nonoccupationally exposed population was in the acceptable range $(<5.0 \mu \mathrm{g} / \mathrm{L})[1]$. The result agreed with the previous report in Thai population [15].

Gender, age, and alcohol consumption were no influences on blood cadmium levels among this study population, similar to the study by Batáriová et al. [16]. Cigarette smoking was found to be significantly related to blood cadmium concentration. Smokers had significantly higher blood cadmium concentration than nonsmokers $(P<0.05)$ (Table 1). It could be explained that cigarette smoking was a major source of nonoccupational cadmium exposure because of cadmium in the tobacco. It had been reported that cigarettes contain cadmium in concentrations ranging from 1.56 to $1.96 \mu \mathrm{g} /$ cigarette [17]. Blood cadmium levels observed in this study group appeared to be similar to those reported in the other studies [15, 16, 18, 19].

Polymorphisms in genes, which involved in metabolism of cadmium, were analyzed including GSTT1, GSTM1, and GSTP1 to explore the genetic susceptibility to cadmium toxicity. In the present study, all tested genotype frequencies were in Hardy-Weinberg equilibrium. The GSTT1 present was the most prevalent genotype found in study population (67.3\%) (Table 2). Homozygous variant of GSTP1 Val/Val was the lowest genotype in study population $(5.1 \%)$. The null genotypes of GSTT1 and GSTM1 were found with a frequency of $32.7 \%$, and $57.6 \%$, respectively, while GSTP1 Ile/Ile, Ile/Val, Val/Val genotype frequencies were $57.3 \%$, $37.6 \%$, and $5.1 \%$, respectively. The similar genotype frequencies were reported in Thai population [20-22].

The GST gene family is involved in the detoxification of electrophilic compounds by conjugating them with GSH and immediately excreted via the bile or urine. The level of GST expression is a crucial factor determining the sensitivity of cell to toxic chemical [23]. This gene family comprises several genes, and many of them are polymorphic in human. GSTP1 exhibits a missense mutation at codon 105, which substitutes Val to Ile. The polymorphism in GSTP1 both amino acid exchanges are in the active site of the enzyme and influence the activity toward different substrates [24]. GSTM1 and GSTT1 exhibit deletion alleles, which eliminate enzyme activity [25]. In the present study, subjects with the GSTP1 homozygous variant (Val/Val) had significantly higher blood cadmium level than did those with Ile/Val and wild-type (Ile/Ile) genotype. The possible explanation would be the substitution of Ile $\rightarrow$ Val in GSTP1 change catalytic activity of the corresponding GSTs $[25,26]$. The change in catalytic activity may reduce in Cd-GSH conjugates and excretion, which results in cadmium accumulated in blood. Since the variation in GSTP1 genotype is associated with variation of blood cadmium concentration. Therefore, it could be assumed that GSTP1 play a more important role in variation of blood cadmium concentrations than the other investigated GST polymorphisms.

With regards to gene-gene interactions, the study showed that GSTP1 Val variant allele had significantly associated with higher blood cadmium concentration when taking into account GSTT1 null or GSTM1 null genotypes (Table 3). This could be explained by GST conjugated reactive compounds to GSH prior to their excretion from the body. The deletion of GSTT1 and GSTM1 genes reduces the catalytic activity [23], and polymorphism in GSTP1, changes catalytic activity of the corresponding GSTs [26]. Therefore, the polymorphisms may affect the conjugation and excretion of cadmium, which results in accumulation of this metal in blood. Moreover, not only a single genetic polymorphism of GSTs genes which may alter the enzyme activity, but also combinations of polymorphism may be more important in determining catalytic activities, expression of enzyme, and interindividual variability of disposition and response to 
substrates. Therefore, this study indicated that the interaction of GSTP1 Val variant allele with GSTT1 null or GSTM1 null could affect the elevation of blood cadmium concentration.

\section{Conclusion}

These results suggested that polymorphisms of GSTP1 and combined genotypes of GSTP1 with GSTT1 and GSTM1 may be responsible for susceptibility to cadmium toxicity. The finding confirms that interindividual variations in blood cadmium concentrations are not entirely attributable to environmental exposure but also genetic background. From the point of view of toxicogenetics, some information of gene-environment and gene-gene interactions is provided by our findings. Further studies are needed to investigate the GSTT1, GSTM1, and GSTP1 mutations and their enzyme activities related to cadmium concentrations. Moreover, genetic variations of GSTs and metallothionein in environmentally and occupationally exposed individuals should be considered, particularly in respect of mechanism of Cdinduced toxicity.

\section{Acknowledgments}

This work was supported by the Cooperative Research Network (CRN) scholarship, the project for Higher Education Research Promotion and National Research University Development, Office of the Higher Education Commission, Ministry of Education, Thailand, and The Thailand Research Fund. The authors wish to thank the EGAT and their people for participating and establishing this study. They would like to express thanks to all research staffs, especially Ms. Nisakron Thongmung and Ms. Yupin Wisetpanit, Office of Research Academic and Innovation, Faculty of Medicine Ramathibodi Hospital, for providing subjective data and technical assistance in specimen collection and preparation.

\section{References}

[1] C. D. Klaassen, Ed., Casarett and Doull's Toxicology: The Basic Science of Poisons, McGraw-Hill, New York, NY, USA, 7th edition, 2008.

[2] M. Valko, H. Morris, and M. T. D. Cronin, "Metals, toxicity and oxidative stress," Current Medicinal Chemistry, vol. 12, no. 10, pp. 1161-1208, 2005.

[3] C. D. Klaassen, J. Liu, and S. Choudhuri, "Metallothionein: an intracellular protein to protect against cadmium toxicity," Annual Review of Pharmacology and Toxicology, vol. 39, pp. 267-294, 1999.

[4] T. Jin, J. Lu, and M. Nordberg, "Toxicokinetics and biochemistry of cadmium with special emphasis on the role of metallothionein," NeuroToxicology, vol. 19, no. 4-5, pp. 529536, 1998.

[5] A. Rossini, D. C. M. Rapozo, L. M. F. Amorim et al., "Frequencies of GSTM1, GSTT1, and GSTP1 polymorphisms in a Brazilian population," Genetics and Molecular Research, vol. 1, no. 3, pp. 233-240, 2002.

[6] N. Ballatori, "Transport of toxic metals by molecular mimicry," Environmental Health Perspectives, vol. 110, no. 5, pp. 689 694, 2002.
[7] R. C. Strange, P. W. Jones, and A. A. Fryer, "Glutathione Stransferase: genetics and role in toxicology," Toxicology Letters, vol. 112-113, pp. 357-363, 2000.

[8] H. Autrup, "Genetic polymorphisms in human xenobiotica metabolizing enzymes as susceptibility factors in toxic response," Mutation Research, vol. 464, no. 1, pp. 65-76, 2000.

[9] C. Gundacker, G. Komarnicki, P. Jagiello et al., "GlutathioneS-transferase polymorphism, metallothionein expression, and mercury levels among students in Austria," Science of the Total Environment, vol. 385, no. 1-3, pp. 37-47, 2007.

[10] H. B. Ketelslegers, R. W. H. Gottschalk, G. Koppen et al., "Multiplex genotyping as a biomarker for susceptibility to carcinogenic exposure in the FLEHS biomonitoring study," Cancer Epidemiology Biomarkers and Prevention, vol. 17, no. 8, pp. 1902-1912, 2008.

[11] P. Sritara, S. Cheepudomwit, N. Chapman et al., "Twelve-year changes in vascular risk factors and their associations with mortality in a cohort of 3499 Thais: The Electricity Generating Authority of Thailand study," International Journal of Epidemiology, vol. 32, no. 3, pp. 461-468, 2003.

[12] K. S. Subramanian and J. C. Meranger, "A rapid electrothermal atomic absorption spectrophotometric method for cadmium and lead in human whole blood," Clinical Chemistry, vol. 27, no. 11, pp. 1866-1871, 1981.

[13] S. A. Miller, D. D. Dykes, and H. F. Polesky, "A simple salting out procedure for extracting DNA from human nucleated cells,” Nucleic Acids Research, vol. 16, no. 3, p. 1215, 1988.

[14] B. R. Packer, M. Yeager, B. Staats et al., "SNP500Cancer: a public resource for sequence validation and assay development for genetic variation in candidate genes," Nucleic Acids Research, vol. 32, no. Database Issue, pp. D528-D32, 2004.

[15] J. Sirivarasai, S. Kaojaren, W. Wananukul, and P. Srisomerang, "Non-occupational determinants of cadmium and lead in blood and urine among a general population in Thailand," The Southeast Asian Journal of Tropical Medicine and Public Health, vol. 33, no. 1, pp. 180-187, 2002.

[16] A. Batáriová, V. Spěváčková, B. Beneš, M. Čejchanová, J. Šmíd, and M. Černá, "Blood and urine levels of $\mathrm{Pb}, \mathrm{Cd}$ and $\mathrm{Hg}$ in the general population of the Czech Republic and proposed reference values," International Journal of Hygiene and Environmental Health, vol. 209, no. 4, pp. 359-366, 2006.

[17] O. Cekic, "Effect of cigarette smoking on copper, lead, and cadmium accumulation in human lens," British Journal of Ophthalmology, vol. 82, no. 2, pp. 186-188, 1998.

[18] L. Farzin, M. Amiri, H. Shams, M. A. Ahmadi Faghih, and M. E. Moassesi, "Blood levels of lead, cadmium, and mercury in residents of Tehran," Biological Trace Element Research, vol. 123, no. 1-3, pp. 14-26, 2008.

[19] N. S. Kim and B. K. Lee, "National estimates of blood lead, cadmium, and mercury levels in the Korean general adult population," International Archives of Occupational and Environmental Health, vol. 84, no. 1, pp. 53-63, 2011.

[20] S. Pakakasama, E. Mukda, W. Sasanakul et al., "Polymorphisms of drug-metabolizing enzymes and risk of childhood acute lymphoblastic leukemia," American Journal of Hematology, vol. 79, no. 3, pp. 202-205, 2005.

[21] P. Pisani, P. Srivatanakul, J. Randerson-Moor et al., "GSTM1 and CYP1A1 polymorphisms, tobacco, air pollution, and lung cancer: a study in rural Thailand," Cancer Epidemiology Biomarkers and Prevention, vol. 15, no. 4, pp. 667-674, 2006.

[22] J. Gatedee, S. Pakakassama, S. Muangman, and W. Pongstaporn, "Glutathione S-transferase P1 genotypes, genetic susceptibility and outcome of therapy in thai childhood acute 
lymphoblastic leukemia," Asian Pacific Journal of Cancer Prevention, vol. 8, no. 2, pp. 294-296, 2007.

[23] J. D. Hayes and R. C. Strange, "Glutathione S-transferase polymorphisms and their biological consequences," Pharmacology, vol. 61, no. 3, pp. 154-166, 2000.

[24] K. S. Engström, U. Strömberg, T. Lundh et al., "Genetic variation in glutathione-related genes and body burden of methylmercury," Environmental Health Perspectives, vol. 116, no. 6, pp. 734-739, 2008.

[25] H. M. Custodio, K. Broberg, M. Wennberg et al., "Polymorphisms in glutathione-related genes affect methylmercury retention," Archives of Environmental Health, vol. 59, no. 11, pp. 588-595, 2004.

[26] M. Dušinská, A. Ficek, A. Horská et al., "Glutathione Stransferase polymorphisms influence the level of oxidative DNA damage and antioxidant protection in humans," Mutation Research, vol. 482, no. 1-2, pp. 47-55, 2001. 

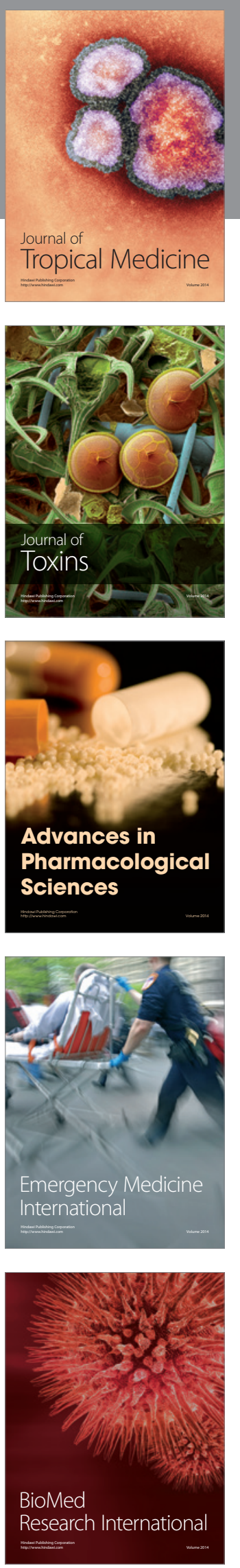
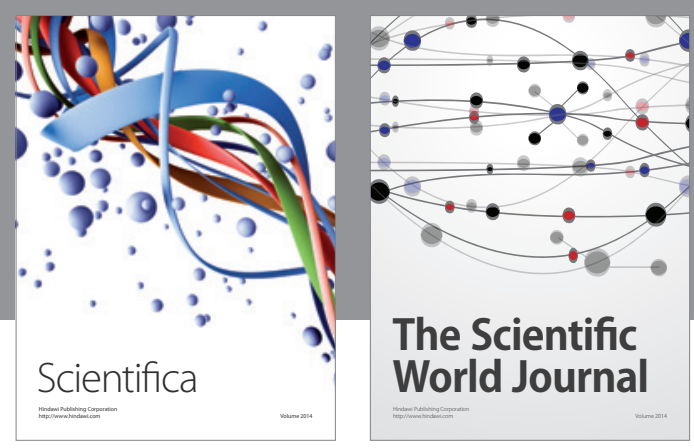

The Scientific World Journal
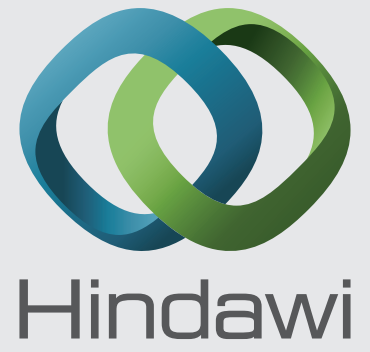

Submit your manuscripts at

http://www.hindawi.com
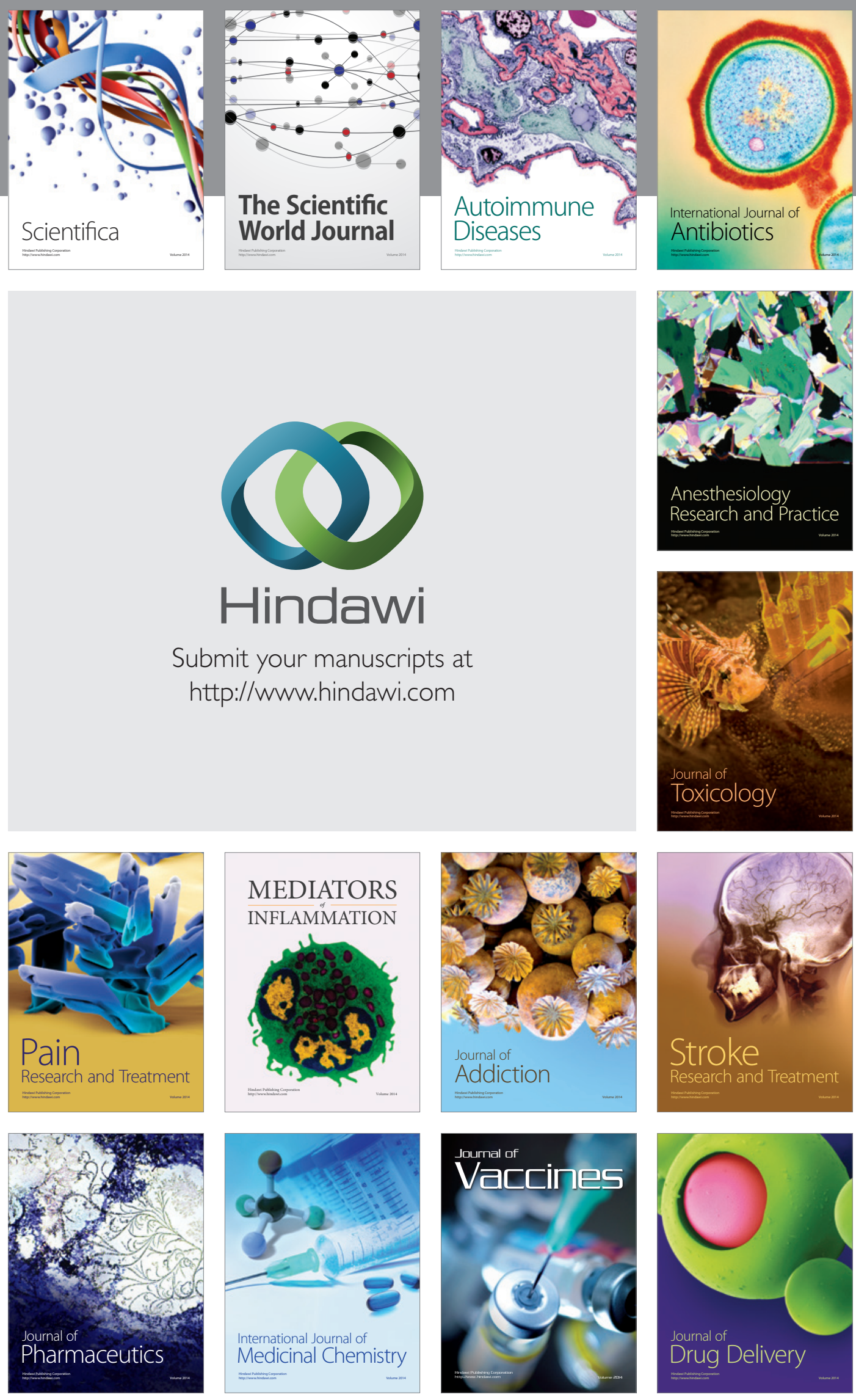